\title{
Flow of colloidal suspensions through small orifices
}

\author{
R. C. Hidalgo* \\ Departamento de Física y Matemática Aplicada, Facultad de Ciencias, and Universidad de Navarra, 31080 Pamplona, Spain \\ A. Goñi-Arana and A. Hernández-Puerta \\ Departamento de Ingeniería Biomédica (TECNUN), Universidad de Navarra, 20009 San Sebastian, Spain \\ I. Pagonabarraga \\ Departament de Física Fonamental, Carrer Martí i Franqués 1, Universitat de Barcelona, 08028 Barcelona, Spain
}

(Received 27 July 2017; revised manuscript received 28 December 2017; published 23 January 2018)

\begin{abstract}
In this work, we numerically study a dense colloidal suspension flowing through a small outlet driven by a pressure drop using lattice-Boltzmann methods. This system shows intermittent flow regimes that precede clogging events. Several pieces of evidence suggest that the temperature controls the dynamic state of the system when the driving force and the aperture size are fixed. When the temperature is low, the suspension's flow can be interrupted during long time periods, which can be even two orders of magnitude larger than the system's characteristic time (Stokes). We also find that strong thermal noise does not allow the formation of stable aggregate structures avoiding extreme clogging events, but, at the same time, it randomizes the particle trajectories and disturbs the advective particle flow through the aperture. Moreover, examining the particle velocity statistics, we obtain that in the plane normal to the pressure drop the colloids always move as free particles regardless of the temperature value. In the pressure drop direction, at high temperature the colloids experience a simple balance between advective and diffusive transport, but at low temperature the nature of the flow is much more complex, correlating with the occurrence of very long clogging events.
\end{abstract}

DOI: 10.1103/PhysRevE.97.012611

\section{INTRODUCTION}

Colloidal suspensions are dispersions of solid particles or droplets embedded in a liquid with different density [1]. They are very common in nature [2], as well as in technological applications $[3,4]$. Thus, it is a significant technological challenge to quantify and predict their permeability through membranes and rheological response in confined geometries [1].

Ten years ago, clogging events were detected experimentally in a colloidal suspension passing through small apertures [5-7]. These authors measured macroscopic variables such as the filtrate flux, the pressure drop, and changes in the volume fraction [5-7]. More importantly, the direct visualization of microparticles or drops has made it possible to obtain a detailed description of the clogs. For instance, in Ref. [8] the clogging of suspensions of monodisperse polystyrene beads in single-pore microchannels was investigated in detail. In that length scale, it was found that clogging occurs regardless of the volumetric flow rate and the suspension's volume fraction [8]. Nevertheless, again at the level of a single pore, Dersoir et al. [9] recently identified two clogging regimes depending on the applied pressure. At low pressure, the clogging time seems to be constant. For higher pressure drops, however, the duration of the clogs depends on the pressure drop. Here, it is important to mention that Sharp and Adrian [10] also observed arching of

\footnotetext{
*raulcruz@unav.es
}

polystyrene particles in microchannels, at a very low volume fraction $(0.5-0.6 \%)$. Explaining their outcomes, they assumed a simple balance between hydrodynamic and contact frictional forces and a weak impact of the surface attraction.

On the other hand, Hong and co-workers [11] investigated experimentally the flow of a quasi-two-dimensional emulsion through a constricting hopper shape. As a result, they found that the suspension flow changes its nature depending on the volumetric flow rate. At low flow rates, the droplets exit the hopper via intermittent avalanches, while at high flow rates the droplets exit continuously. Moreover, the same group studied experimentally the flow of oil-in-water emulsion droplets through a quasi-two-dimensional hopper [11], obtaining that clogging is only produced with a very narrow hopper opening. Those results contrast with previous studies with frictional disks, where arch formation and clogging were detected with significantly larger hopper openings [12-15]. In this regard, upon exploring low-adherence regimes in microfluidic devices, the authors of [16-18] determined that non-Brownian polystyrene particles behaved very similar to dry granular systems when flowing through very small orifices. Additionally, it is worth mentioning that recent experimental works have shown that the rheological responses of colloids and emulsions are very sensitive to the confining conditions [19-22].

Some theoretical and numerical efforts have been made in the field of colloid release and clogging [23-30]. For instance, recently Agbangla et al. [26] analyzed numerically the collective hydrodynamic and colloidal effects of microparticles mov- 
ing through a pore. They found very stable clogged structures induced by strong particle-particle interaction and particlewall adhesion, which prevents rearrangements of particles within an aggregate and enhances clog stability. Moreover, very detailed theoretical analyses have recently enabled us to describe clog formation [25-30]. Thus, it is currently accepted that the clogging process is mainly ruled by the driven force acting on the particles, long-range hydrodynamic interactions, and the nature of the particle-particle and particle-wall interactions [23-30].

It is a fact that in colloidal suspensions, both rheological properties and equilibrium phase behavior are very sensitive to changes in temperature. Moreover, flow-stopping blockages in colloidal suspensions are usually controlled by local mechanisms that determine how the aggregate of particles grow and stick to the confining surface. The question is whether those local mechanisms are affected by thermal noise. For instance, it was recently found experimentally that high-frequency and low-amplitude perturbation perpendicular to the shearing direction can unjam flow-induced structures [31]. In our work, we examine numerically the role of thermal fluctuations on colloidal suspensions when flowing through a small orifice, driven by a pressure drop. We find that the thermal fluctuations have a significant influence on the stability of the clogs. The paper is organized as follows: in Sec. II we briefly describe the numerical model. In Sec. III we discuss the numerical results of the particle dynamics crossing the orifice and the statistics of clogging events. Finally, we provide our conclusions and an outlook.

\section{NUMERICAL MODEL}

We examine colloidal suspensions composed of spherical particles of radius $R$ embedded in a liquid. The fluid is modeled using a lattice Boltzmann approach (LB), which resembles the solution of the Navier-Stokes equations at large time scales [32]. At each lattice node, the liquid is defined by a distribution function $f(\vec{r}, t)$ that evolves in discrete time steps as

$$
f_{i}\left(\vec{r}+\vec{c}_{i} \Delta t ; t+\Delta t\right)=f_{i}(\vec{r} ; t)+\Omega_{i j}\left[f_{j}^{\mathrm{eq}}(\vec{r} ; t)-f_{j}(\vec{r} ; t)\right] .
$$

This evolution rule accounts for the linear momentum streaming to the neighboring nodes $j$ due to the liquid advection motion of velocity $\vec{c}_{i}$. Moreover, a collision operator $\Omega_{i j}$ controls the relaxation process toward an equilibrium state, characterized by $f_{j}^{\mathrm{eq}}(\vec{r} ; t)$. Here we use Ludwig, an implementation of the lattice Boltzmann method adapted to the study of complex fluids, and which puts into effect a multirelaxation implementation of the collision operator $\Omega_{i j}$ [33]. The system geometry is a three-dimensional (3D) cubic lattice with 19 allowed velocities $\vec{c}_{i}$ known as a $D_{3} Q_{19}$ scheme [32]. We use units such that the mass of the nodes, the lattice spacing, and the time step are 1 and the kinematic viscosity is $v$.

Thermal fluctuations are included in the lattice Boltzmann model adding an additional noise term to the left of Eq. (1), $\xi_{i}$. This additional contribution introduces fluctuations in the populations in each phase-space cell. The stochastic properties of this term are chosen to ensure that the fluctuation-dissipation theorem in equilibrium is satisfied. Toward that end, the amplitudes of the random sources are linked to the properties of the sources of dissipation of the collision operator. This choice ensures that mass and momentum conservation are preserved by this noise contribution, and that equipartition is satisfied in equilibrium, which guarantees that in the absence of any forcing, the suspension reaches thermal equilibrium [34,35].

The colloids are modeled defining solid particles of radius $R$, which interact with the surrounding fluid through bounceback links. Hence, the total force and torque acting on a solid particle are determined using the mechanical constraint, which is given by the momentum exchange between the solid particle and the surrounding fluid nodes. Additionally, a contact interaction between the colloids was also used. For the sake of simplicity, we have assumed that the interaction between two solid particles is defined by the potential,

$$
v^{s s}(r)=\epsilon\left(\frac{\sigma}{r}\right)^{v_{o}},
$$

where $\epsilon$ accounts for the strength of the interaction, $\sigma$ is the length scale, and $v_{0}$ characterizes the range of particle-particle interaction. The numerical implementation of the interaction potential Eq. (2) ensures that colloids do not overlap at the hard-core radius $R$ when introducing a so-called hydrodynamic radius $R_{h}$ [36]. In general, simulating multiparticle suspension, all distance calculations are based on the hydrodynamic radius $R_{h}$, and to obtain accurate results it is then essential to use a calibrated value [37], which is typically larger than the physical radius $R$. Thus, we have used $\epsilon=0.04, \sigma=1.0$, $v_{0}=1.0, R=4.8$, and $R_{h}=5$ (in lattice units), which ensure that colloids do not overlap $(\Delta r>0.2)$ in the studied stress conditions [38,39].

In our numerical experiment, the colloidal suspension is confined between two parallel solid walls that have a circular orifice of modifiable radius $W$. Thus, the interaction with the wall is suppressed in the orifice region, which allows the suspension to flow through it (in the $y$ direction). Moreover, while the colloidal particles are passing through the orifice, they interact with an imaginary colloid with radius $R$, which replicates the aperture boundary. In the other two directions $(x$ and $z$ ), the system is periodic.

The system size is 44 nodes wide in the transverse direction and 88 nodes wide in the direction perpendicular to the solid walls. A total of 128 colloidal particles are initially randomly distributed, corresponding to an average volume fraction $\phi=$ 0.4 . The fluid is subject to a uniform force perpendicular to the walls, mimicking a pressure-driven flow that pushes the colloidal particles through the opening. Thus, a constant pressure gradient $\frac{\Delta p}{L}$ is applied in a direction normal to the walls. This forcing induces the accumulation of the colloidal particles in the neighborhood of the orifice. However, long simulations are required to sample appropriately the statistics of clogging events. Toward that end, in each simulation the colloidal particles are allowed to cross the system size several times. After crossing the aperture region at $y=88$ in lattice units, the particles reenter at the same $x$ and $z$ position at which they exited but with $y$ equal to $y-88$ in lattice units [see Fig. 1(a)]. We typically run $T=10^{8}$ time steps. However, in some cases the particle flow is interrupted indefinitely. The simulation are executed several times to gather statistics of at least $10^{5}$ colloidal particles passing through the orifice. 

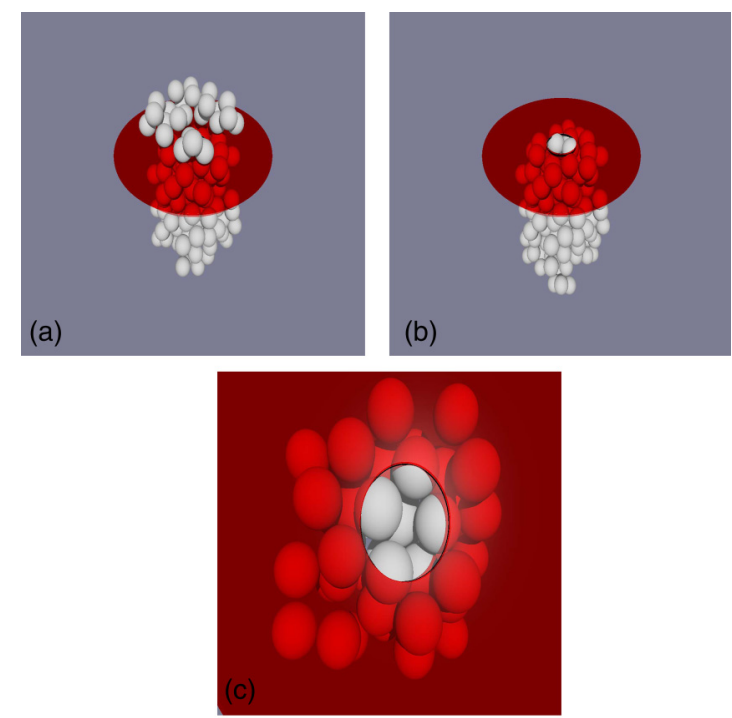

FIG. 1. Part (a) illustrates the simulation box of 3D colloidal suspensions passing through a small circular orifice. Parts (c) (lateral view) and (b) (top view) show a clog as observed in the simulations.

In the present study, attention is focused on the influence of the temperature. An outlet size of radius $W=1.7 \times R$ and a pressure drop of $\frac{\Delta p}{L}=5 \times 10^{-5}$ are fixed. The fluid parameters are chosen to ensure a low Reynolds number $\operatorname{Re}=U_{c} R / v$, which can be estimated using the particle radius $R$, the fluid kinematic viscosity $v=0.5$, and the system characteristic velocity $U_{c}=\frac{W^{2}}{4 v} \frac{\Delta p}{L}$. In the following sections, the values of $k_{B} T$ are given in terms of the colloid's characteristic kinetic energy $E_{c}=\frac{m U_{c}^{2}}{2}$, where $m$ is the mass of the colloid. In all cases, the Reynolds number Re results smaller than 0.02 .

\section{RESULTS AND DISCUSSION}

Initially, the colloidal particles are randomly distributed in the space domain, and the pressure drop induces a flow. As time passes, particles flow continuously through the orifice, experiencing a driven motion in the $y$ direction while moving diffusively in the $x$ and $z$ directions [see Fig. 1(a)]. Furthermore, the interaction with the orifice edge induces flow-rate fluctuations, and, eventually, clogging events are detected. In Figs. 1(c) (lateral view) and 1(b) (top view), we illustrate a vault that is typically formed at the orifice perturbing the global flow. In general, both the structure formed and its stability are nontrivial, and they depend on the pressure gradient, the temperature, and the orifice size.

The simulations show that temperature plays an important role in the stability of large macroscopic clogging events at the orifice. Figure 2 illustrates the time-series data of clogging times $\Delta t$ detected during the flowing processes. For comparison, results corresponding to three different temperatures $T$ are displayed. Additionally, in each case we estimated the values of the Peclet number, defined as the ratio between the mean advective $S_{\text {adv }}$ and diffusive $S_{\text {diff }}$ displacements of the particle in the unit of LB time, yielding $\mathrm{Pe}=\frac{S_{\mathrm{adv}}}{S_{\mathrm{diff}}}=\frac{U_{c}}{\sqrt{D}}=\sqrt{\frac{6 \pi \nu R}{k_{B} T}} U_{c}$.

The clogging time $\Delta t$ is defined as the time lapse between the passing of two consecutive particles through the orifice.
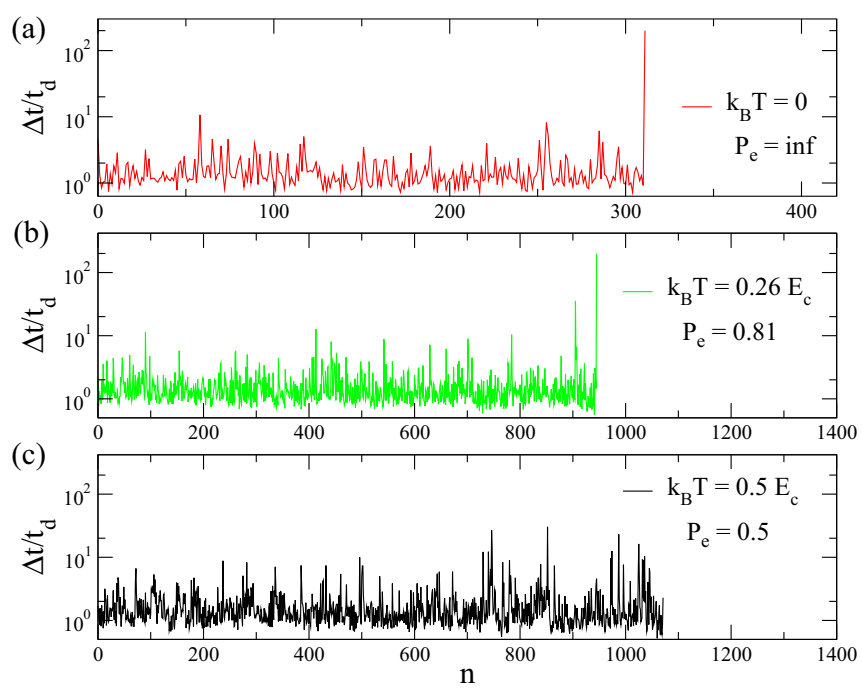

FIG. 2. Time-series data of clogging times $\Delta t$ during the flowing process rescaled with the characteristic time $t_{d}$. Data correspond to temperatures: (a) $T=0$, (b) $T=0.26 E_{c}$ below, and (c) $T=0.50 E_{c}$ above the clogging transition.

Besides that, $n$ represents the index of the crossing particle. Note that the values of $\Delta t$ are normalized with the characteristic time $t_{d}$, in which the colloid moves the length of its own diameter, driven by the pressure drop without interacting with the orifice and with other particles. This value is estimated as $t_{d}=\frac{2 R}{U_{c}} \approx 5 \times 10^{3}$. Thus, from Fig. 2 one can conclude that even though the pressure gradient continually drives the colloids to move through the aperture, the formation of particle structures is frequently detected. When $T=0$ [see Fig. 2(a)], particle aggregates totally block the orifice just after a few particles have passed through it, and consequently the particle flow is interrupted indefinitely. Note that, for practical reasons, we have defined an infinite clogging time when $\Delta t>200 t_{d}=$ 1000000 steps. When introducing thermal fluctuations, however, the formation of stable aggregate structures is less likely, delaying the occurrence of events with infinite clogging time [see Figs. 2(b) and 2(c)]. Note that for the case of very strong thermal fluctuations [see Fig. 2(c)], permanent particle flow interruptions are not detected.

One can gain insight into the colloid-current fluctuations by examining the burst size $s=\Delta n$, i.e., the number of colloids crossing the orifice between two macroscopic clogging events. However, it is arbitrary in principle to define the size of a macroscopic clogging event $\Delta t_{c}$. For a better view and discussion, we have defined $\Delta t_{c}$ in terms of the characteristic time $t_{d}$. In Fig. 3, the probability density distributions $D(s)$ are illustrated. For comparison, we illustrate the burst statistics using two different definitions $\Delta t_{c}=t_{d}$ and $4 t_{d}$. Note that in both cases the data are in agreement with an exponential behavior, which suggests that the probability of clogging is constant during the burst. Notably, similar results were observed in very different scenarios when exploring pedestrians [40] and flocks [41] passing through a narrow door.

In Fig. 4 we study the evolution of the mean burst size $\langle s\rangle$ with $\Delta t_{c}$. As is expected, in all cases $\langle s\rangle$ grows monotonously with $\Delta t_{c}$. For $\Delta t_{c} \geq 5 t_{d}$, we found that the increase of $\langle s\rangle$ is 

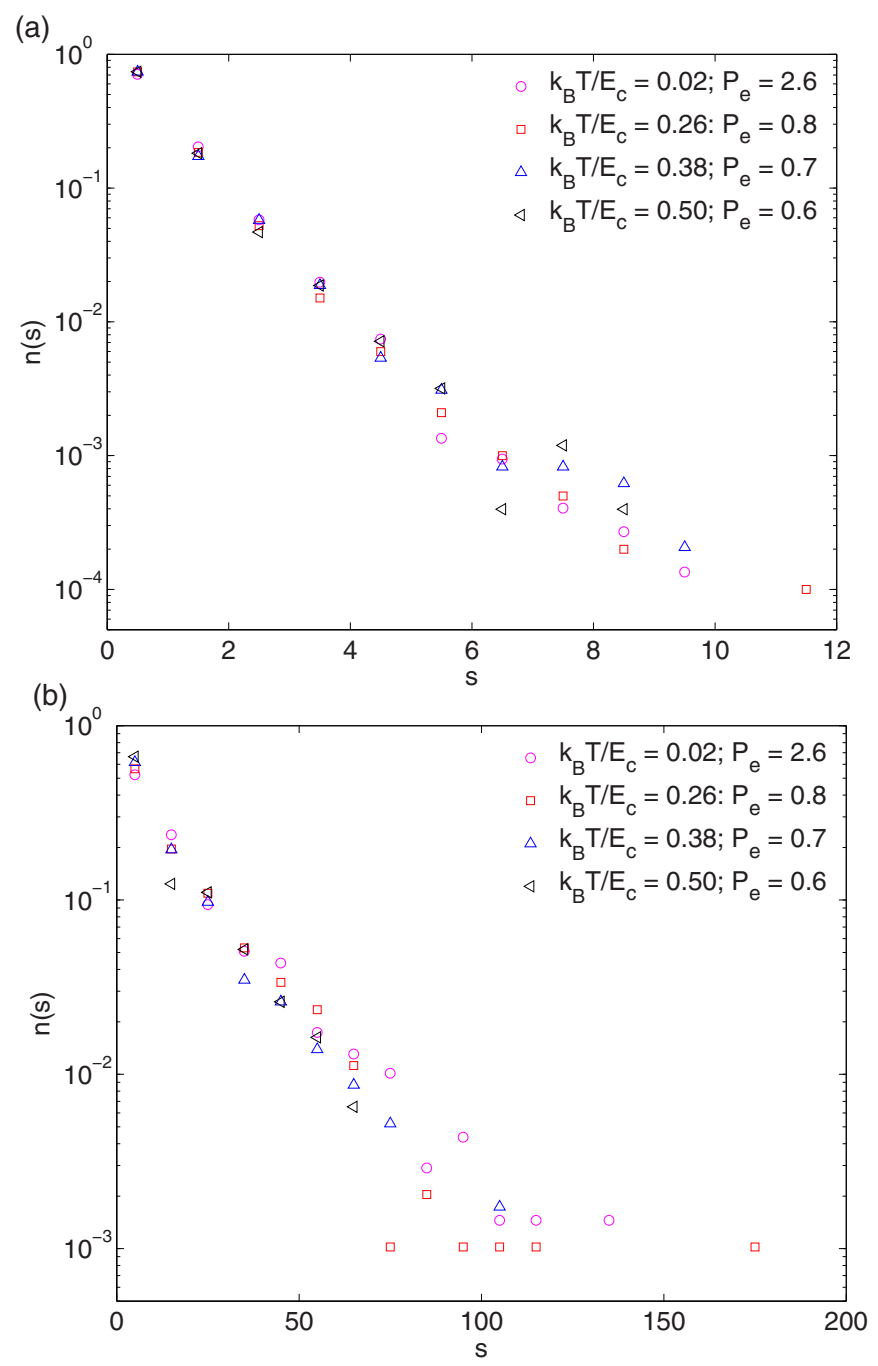

FIG. 3. Probability density distributions of the burst size. For the definition of a burst, the persistence of macroscopic clogging events of (a) $\Delta t_{c}=t_{d}$ and (b) $\Delta t_{c}=4 \times t_{d}$ was used.

faster as the temperature is lower, denoting that the number of colloids crossing the orifice between consecutive extreme events of size $\Delta t_{c}$ decreases with increasing temperature. The latter suggests that the thermal fluctuations prevent the pressure-driven particle flow through the orifice. This finding contrasts with previous experimental results in vertical vibrated silos, in which arch destabilization and an increase in the flow rate were induced by increasing the vibration amplitude $[14,42]$. However, this difference might be related to the fact that thermal fluctuations are isotropic and that the orifice and the particle size are very close. Very recently, To and co-workers reported the flow in vibrated silos in which the orifice location oscillates in the horizontal direction [43]. They found that when the orifice size and the particle ratio are very similar, the vibration hinders the entrance of the particles through the orifice. As a result, the macroscopic flow rate decreases with increasing oscillation amplitude. In our case, we speculate that thermal fluctuations induce a locking effect when a single particle is crossing the aperture and it interacts with the aperture border.

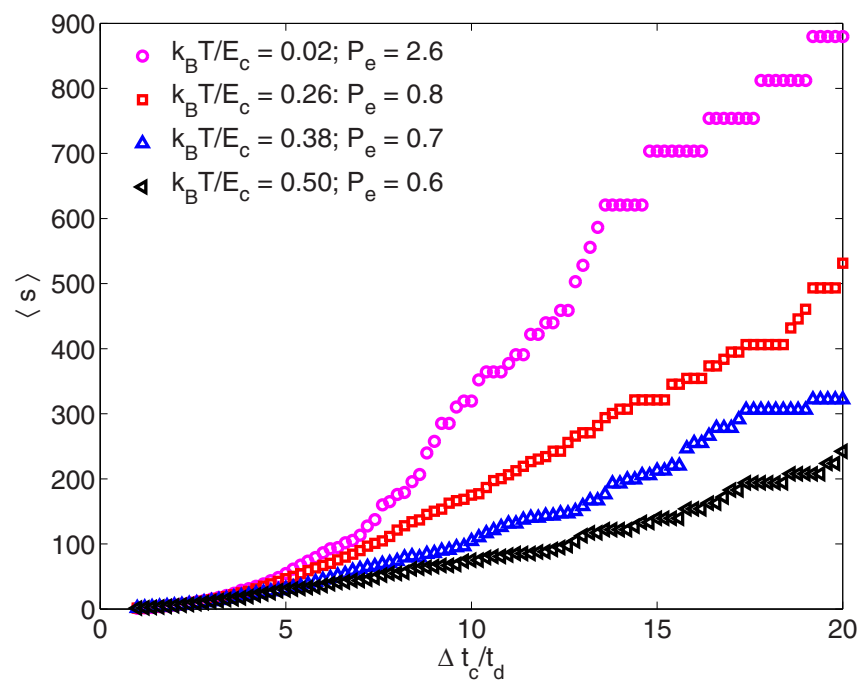

FIG. 4. Mean burst size $\langle s\rangle$ as a function of the value $t_{c}$ chosen for the definition of clogs.

The statistics of the clogging times $\Delta t$ reveals notable features of the clogging process. The probability density distributions $D\left(\Delta t / t_{d}\right)$ and their complementary cumulative distributions $P\left(t>\Delta t / t_{d}\right)$ are illustrated in Fig. 5. Outcomes obtained in systems with different temperatures are compared. The first issue is the detection of very fast events $\left(\Delta t<t_{d}\right)$. Moreover, they are more likely when the temperature is high (low Pe values), which indicates that they correlate with strong thermal fluctuations acting in the direction of the flow. For low temperature, however, those events are rare.

Examining the probability density distributions $D\left(\Delta t / t_{d}\right)$, it is hard to elucidate the effect of thermal fluctuations on the statistics of long clogging times. To look deeply into the nature of these distributions, we also compute the complementary cumulative distributions [see Fig. 5(b)]. Remarkably, the statistics of extreme $\Delta t / t_{d}$ values is affected by temperature changes. In the limit of very low temperature $(\mathrm{Pe}=\infty)$, we observe that the tails of the cumulative distributions follow the power law $P\left(t>\Delta t / t_{d}\right)=\Delta t^{-\alpha}$ with $\alpha<2$. As the temperature is increased, however, the occurrence of long clogs is less likely, and we obtain that the value of $\alpha$ increases continuously. In the limit of very high temperature $(\mathrm{Pe}=0.6)$, the cumulative distributions $P\left(t>\Delta t / t_{d}\right)$ compare better with an exponential law. From these results, one can conclude that strong thermal noise does not allow the formation of stable aggregate structures, avoiding the occurrence of extreme clogging events. Our outcomes indicate that there is a well-defined transition, which is detected by changing the temperature. We find that above $T_{c} \approx 0.26 \frac{m U_{c}^{2}}{2 k_{B}}$ the tail of the cumulative distribution fits a power-law function with $\alpha<2$, which corresponds to clogged states characterized by an infinite value of the mean clogging time $\langle\Delta t\rangle=\infty$. We refer to that state $T>T_{c}$ as a clogged state [44]. For $T<T_{c}$, however, the exponent result $\alpha>2$, i.e., the probability of detecting very long clogs, is notably lower and the flow states are characterized by a finite mean clogging time (unclogged state) [44].

Interestingly, we found that the values of the Peclet number $\mathrm{Pe}=\frac{S_{\mathrm{adv}}}{S_{\mathrm{diff}}}$ are within the range $\mathrm{Pe}=2.6$ (the lowest 


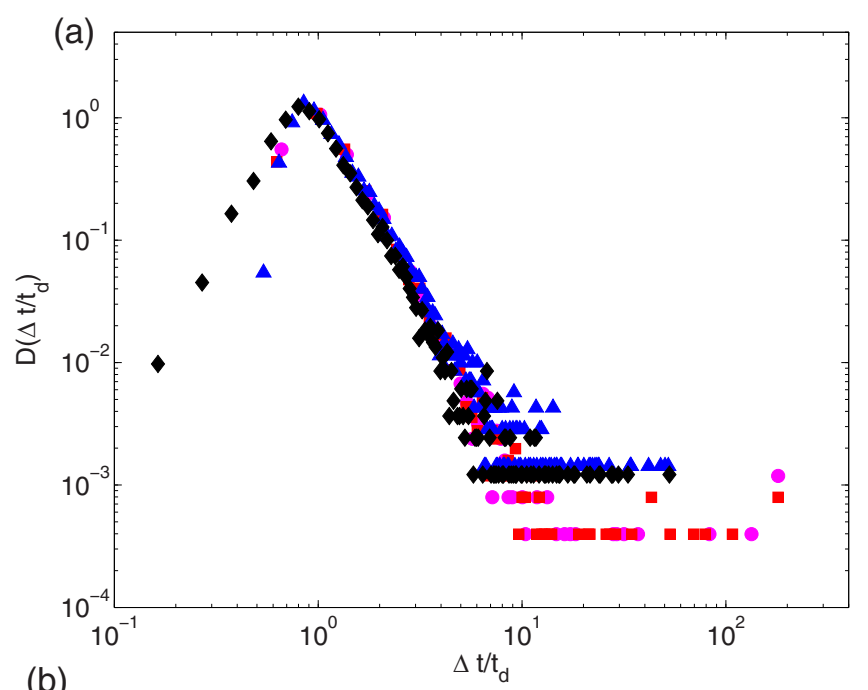

(a)
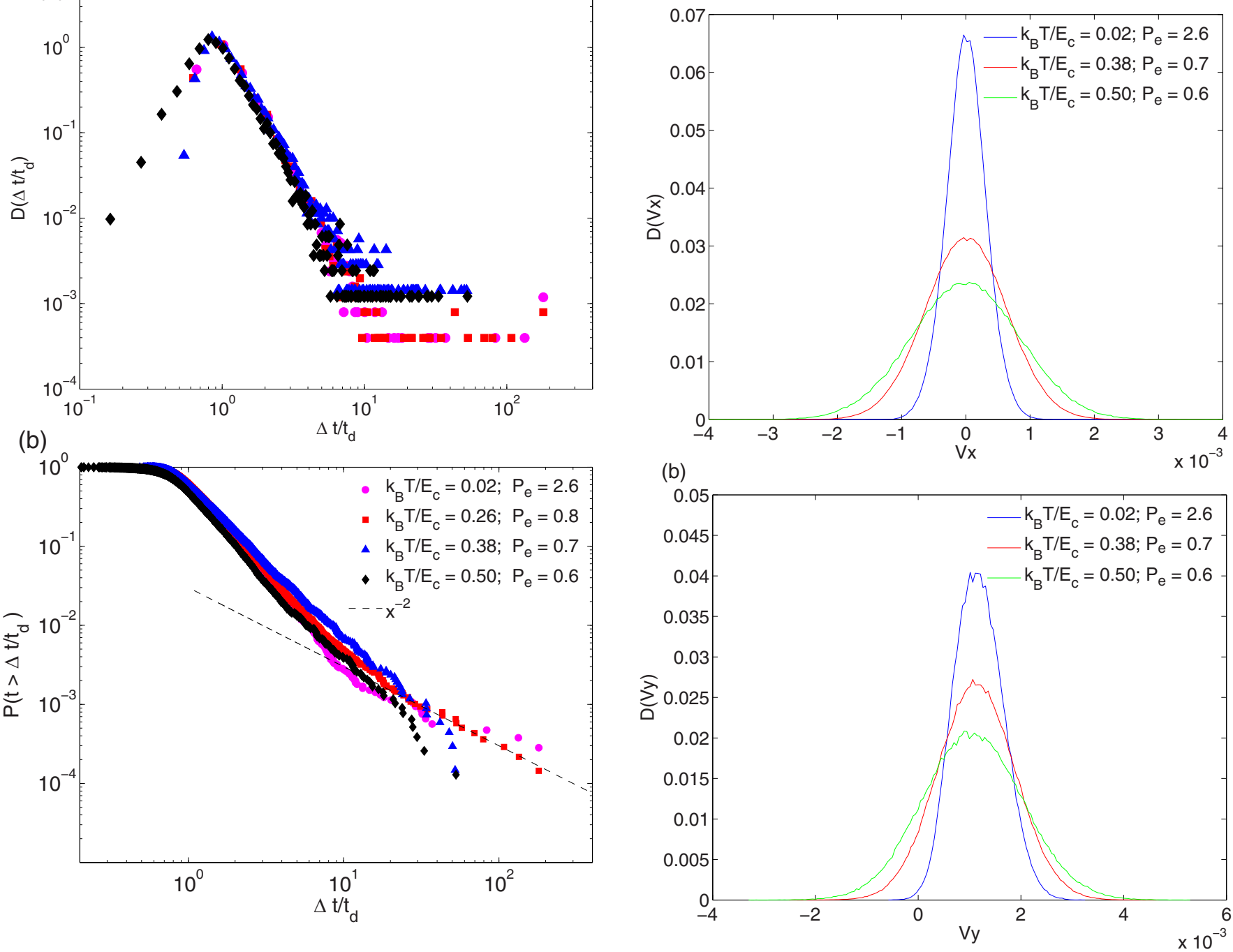

FIG. 5. (a) Probability density and (b) cumulative distribution functions of the time lapses obtained for an outlet with radius $W=1.7 \times R$ and different temperatures (in units of the colloid characteristic kinetic energy, as indicated in the legend).

temperature) and $\mathrm{Pe}=0.6$ (the highest temperature). These results suggest that the thermal bath notably randomizes the particle trajectories over the unit of time. This correlates with the fact that the number of colloids crossing the orifice between consecutive extreme events of size $\Delta t_{c}$ decreases with increasing temperature (see Fig. 4). Thus, large thermal fluctuations disturb the advective particle flow through the aperture, resulting in a notably lower Peclet value. Controversially, the statistics of clogging times also indicates that strong thermal fluctuations are significant in preventing extremely long clogging events (see Fig. 5). This finding indicates that thermal fluctuations do not allow the development of very stable structures, which are responsible for the occurrence of those extreme events.

Additionally, the velocity statistics of the colloids is also examined for different temperatures. The velocity of each colloid is recorded when crossing the orifice, and the probability densities $D(v)$ are computed for each velocity component. The data obtained for three temperatures are illustrated in Fig. 6.

FIG. 6. Velocity distributions obtained in a region close to the aperture (after crossing) for different temperatures. (a) $D\left(V_{x}\right)$ measured perpendicular to the pressure gradient, and (b) $D\left(V_{y}\right)$ measured parallel to the pressure gradient.

Note that no driven force is acting on the $x$ and $z$ directions, and, as a result, the probability distributions are Gaussian centered at $v_{x}=0$ and $v_{z}=0$ (data not shown). In fact, in the $x-z$ plane the colloids behave as free particles moving diffusively, with equal probability in any direction. As expected, the width of the curve is proportional to the intensity of thermal vibrations. On the other hand, the probability distribution of $v_{y}$ is shifted toward the positive direction as a consequence of the liquid advection. Thus, in the $y$ direction the particles are transported by the fluid, while their individual movement fluctuates due to the thermal noise.

To clarify the impact of both advective and diffusive transport, we carefully check the symmetry of the probability density distributions. Toward that end, we subtract the advective velocity $\left\langle v_{y}\right\rangle$ from the velocity of each particle $v_{y}$, and we calculate the probability density distribution with respect to the moving reference frame $D\left(v_{y}-\left\langle v_{y}\right\rangle\right)=$ $D\left(V_{r}\right)$. For comparison, in Fig. 7 the distributions $D\left(V_{r}\right)$ and 
(a)

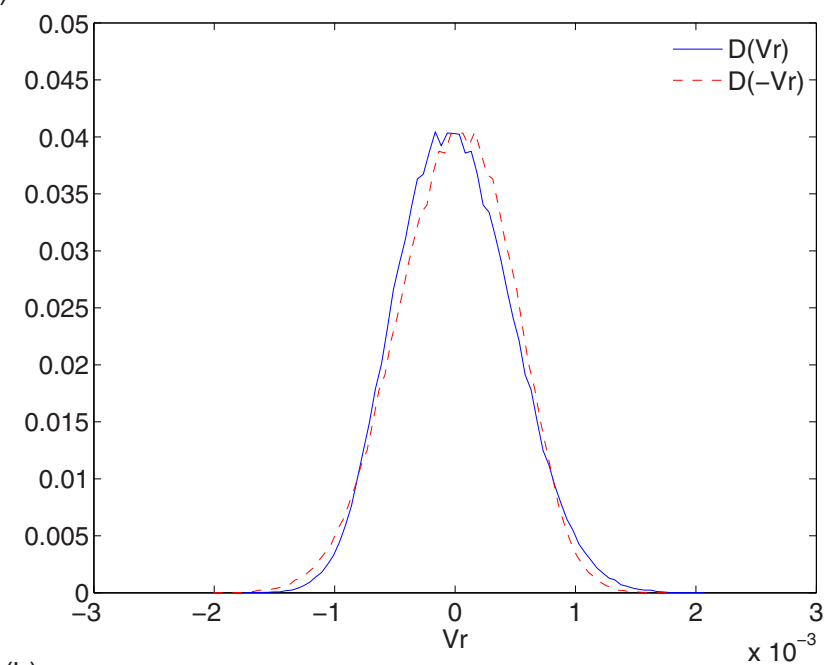

(b)

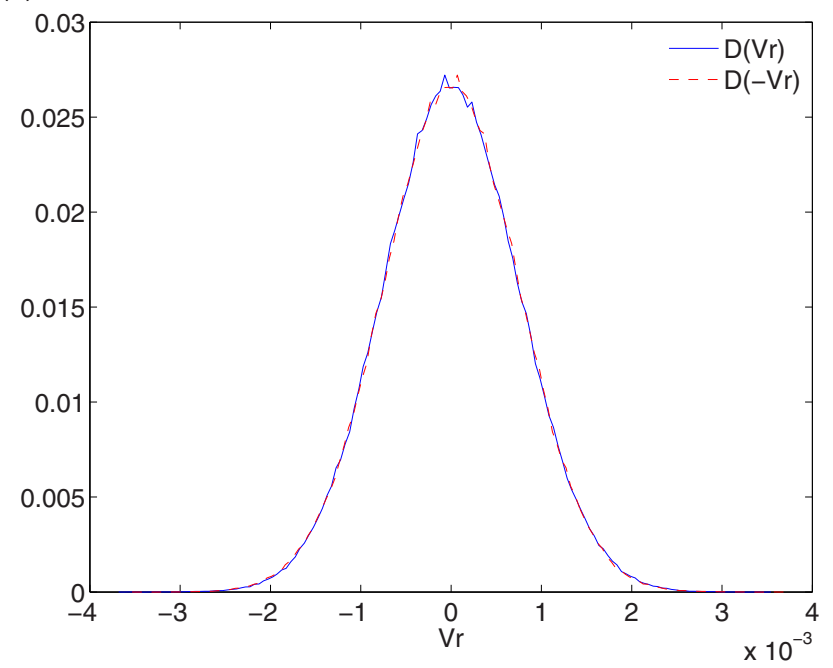

FIG. 7. Velocity distribution $D\left(V_{r}\right)$, measured in a direction parallel to the pressure gradient, with respect to a reference system $V_{r}=$ $v_{y}-\left\langle v_{y}\right\rangle$ in (a) results corresponding to $T<T_{c}$ (clogged region) and (b) results corresponding to $T>T_{c}$ (unclogged region).

$D\left(-V_{r}\right)$ are illustrated. It is noticeable that systems with high temperature $T>T_{c}$ exhibit very symmetric probability density distributions indicating a simple balance between advective and diffusive transport through the orifice. In contrast, for $T<T_{c}$ the curves do not collapse, suggesting that the flow nature is much more complex than a simple superposition of advective and diffusive motions. This fact correlates with the development of very stable structures and very long clog events. This analysis is significant because it provides a tool with which to examine the nature of suspension flow through bottlenecks.

Examining the time evolution of macroscopic properties, we also capture interesting features of the clogging process. For instance, the behavior of the aperture permeability for the particles defined as $k(t)=\frac{v \bar{v}_{y}}{\Delta p / L}$ is shown in Fig. 8. The value $\bar{v}_{y}$ accounts for the mean velocity of colloids that cross the aperture in a given time window in the direction perpendicular to the wall. In the description, the magnitude
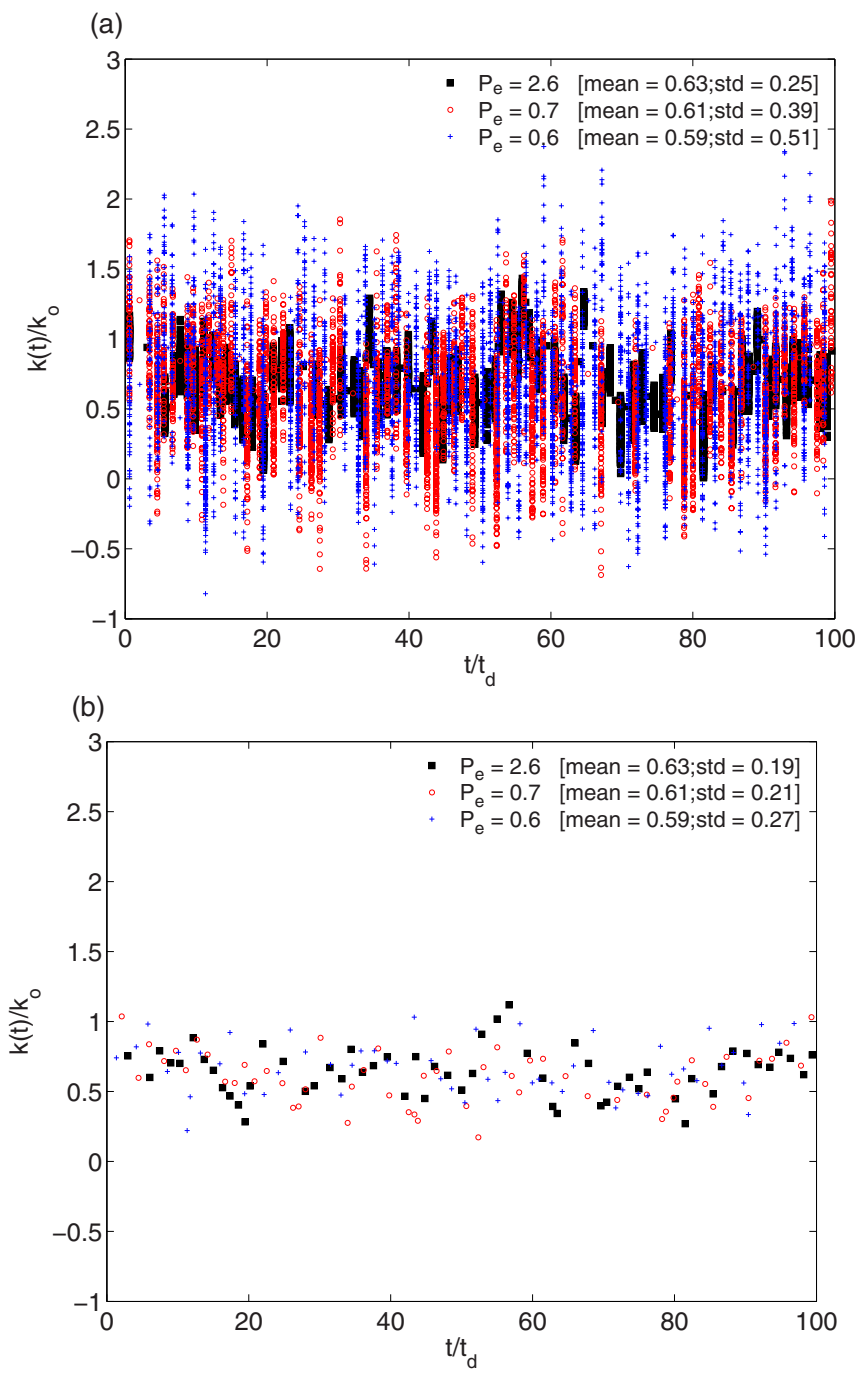

FIG. 8. Behavior of the normalized permeability $\frac{k(t)}{k_{o}}=\frac{\bar{v}_{y}}{U_{o}}$. The values of $\frac{k(t)}{k_{o}}$ are averaged over two time windows (a) one time step and (b) 100 time steps. Outcomes corresponding to different temperatures are shown.

$k(t)$ is normalized using the reference aperture permeability to the fluid, $k_{o}$, which corresponds to the liquid flow through an orifice free of particles, $k_{o}=\frac{\nu U_{o}}{\Delta p / L}$, yielding $\frac{k(t)}{k_{o}}=\frac{\bar{v}_{y}}{U_{o}}$. Note that this magnitude is proportional to the instantaneous current of colloids crossing the orifice. In Figs. 8(a) and 8(b) the values of $\frac{k(t)}{k_{o}}$ are illustrated, corresponding to an average over one time step and 100 time steps, respectively. This procedure enables us to differentiate between thermal fluctuations and fluctuations induced by the presence of the hole. As might be expected, the amplitude of the $\frac{k(t)}{k_{o}}$ fluctuations correlates with the liquid temperature. Note that by increasing the width of the time windows, the instantaneous fluctuations of $\frac{k(t)}{k_{o}}$ decrease toward an asymptotic value, which no longer depends on the temperature [see Fig. 8(b)]. The remaining fluctuations correspond to the limiting case $T=0$. In fact, those remaining fluctuations are only related with the geometrical granular constraint, which is typically governed by the ratio between the orifice size and the colloid's diameter $W / 2 R$. Finally, we find that the mean value of $\frac{k(t)}{k_{o}}$, averaged over a much 
longer period of $100 \times t_{d}$, increases slightly upon increasing the Peclet number Pe (see the mean values in Fig. 8).

\section{CONCLUSIONS}

Summarizing, we present a systematic study of a colloidal suspension flowing through an orifice driven by a pressure drop and with thermal fluctuations. The model allows us to control the temperature, the orifice size, and the pressure drop, but attention is focused here on the significance of the temperature. First, we explore the flow fluctuations by describing the statistics of bursts, which is in agreement with an exponential behavior. The latter suggests that the probability of clogging is constant during the bursts. Moreover, we find that the mean number of colloids crossing the orifice between macroscopic clogging events decreases with increasing temperature, which indicates that thermal fluctuations prevent the particle flow through the orifice. We speculate that the isotropic nature of the thermal fluctuations might induce a locking effect while a single particle crosses the aperture. However, we also found that the thermal fluctuations play an important role in preventing extremely long clogging events, and, as a result, the temperature controls the dynamic state of the system when the driving force is fixed. Thus, for low temperature, we observe that the time elapse distributions display power-law tails $D\left(\Delta t / t_{d}\right)=\Delta t^{-\alpha}$ with $\alpha<2$, which corresponds to a clogged state characterized by the infinite value of the mean clogging time $\langle\Delta t\rangle=\infty$. As the temperature is increased, however, we find that long clogs are less likely to have power-law tails with $\alpha>2$ or exponential laws. This scenario represents flow states with a finite value of the mean clogging time. Remarkably, we observe that the approach to complete obstruction in colloidal suspensions obeys the same universal scenario as granular materials [14], animal herds [41], human models [44], and experiments [45]. Finally, examining the velocity statistics of the particle close to the orifice, for $T>T_{c}$ we observe a simple balance between advective and diffusive transport. In contrast, for $T<T_{c}$ the movement of individual particles is much more complex. This result is significant because it provides a tool to examine the nature of suspension flow through bottlenecks.

Our outcomes lead to several questions on the fundamental mechanisms behind clogging in colloidal suspensions when flowing through small orifices. Our intention is to address some of those questions in future works by exploring the significance of the pressure drop, the orifice diameter, and the balance between hydrodynamic forces and the interaction potential.

\section{ACKNOWLEDGMENTS}

The Spanish MINECO (Projects FIS2014-57325 and FIS2017-84631) has supported this work. I.P. acknowledges DURSI for financial support under the project 2014SGR-922 and Generalitat de Catalunya under Program Icrea Acadèmia. The authors thank D. Maza, I. Zuriguel, and A. Garcimatín for fruitful discussions, and F. Alarcon for his technical support.
[1] J. Mewis and N. J. Wagner, Colloidal Suspension Rheology, Cambridge Series in Chemical Engineering (Cambridge University Press, Cambridge, 2012).

[2] S. Laurie Sanderson, A. Y. Cheer, J. S. Goodrich, J. D. Graziano, and W. Todd Callan, Crossflow filtration in suspension-feeding fishes, Nature (London) 412, 439 (2001).

[3] J. R. Valdes and J. C. Santamarina, Particle transport in a nonuniform flow field: Retardation and clogging, Appl. Phys. Lett. 90, 244101 (2007).

[4] A. Sauret, E. C. Barney, A. Perro, E. Villermaux, H. A. Stone, and E. Dressaire, Clogging by sieving in microchannels: Application to the detection of contaminants in colloidal suspensions, Appl. Phys. Lett. 105, 074101 (2014).

[5] M. D. Haw, Jamming, Two-Fluid Behavior, and "Self-Filtration" in Concentrated Particulate Suspensions, Phys. Rev. Lett. 92, 185506 (2004).

[6] I. Ochoa and S. G. Hatzikiriakos, Paste extrusion of polytetrafluoroethylene (ptfe): Surface tension and viscosity effects, Powder Technol. 153, 108 (2005).

[7] L. Isa, R. Besseling, E. R. Weeks, and W. C. K. Poon, Experimental studies of the flow of concentrated hard sphere suspensions into a constriction, J. Phys.: Conf. Ser. 40, 124 (2006).

[8] H. M. Wyss, D. L. Blair, J. F. Morris, H. A. Stone, and D. A. Weitz, Mechanism for clogging of microchannels, Phys. Rev. E 74, 061402 (2006).

[9] B. Dersoir, M. R. de Saint Vincent M. Abkarian, and H. Tabuteau, Clogging of a single pore by colloidal particles, Microfluid. Nanofluid. 19, 953 (2015).
[10] K. V. Sharp and R. J. Adrian, On flow-blocking particle structures in microtubes, Microfluid. Nanofluid. 1, 376 (2005).

[11] X. Hong, M. Kohne, M. Morrell, H. Wang, and E. R. Weeks, Clogging of soft particles in two-dimensional hoppers, Phys. Rev. E 96, 062605 (2017).

[12] K. To, P. Y. Lai, and H. K. Pak, Jamming of Granular Flow in a Two-Dimensional Hopper, Phys. Rev. Lett. 86, 71 (2001).

[13] I. Zuriguel, A. Garcimartín, D. Maza, L. A. Pugnaloni, and J. M. Pastor, Jamming during the discharge of granular matter from a silo, Phys. Rev. E 71, 051303 (2005).

[14] A. Janda, I. Zuriguel, A. Garcimartín, L. A. Pugnaloni, and D. Maza, Jamming and critical outlet size in the discharge of a two-dimensional silo, Europhys. Lett. 84, 44002 (2008).

[15] J. Koivisto and D. J. Durian, The sands of time run faster near the end, Nat. Commun. 8, 15551 (2017).

[16] A. Marin, H. Lhuissier, M. Rossi, A. Volk, and C. J. Kähler, A fluidic hourglass, http://meetings.aps.org/link/BAPS.2016.DFD.A30.8 (2016).

[17] A. Marin, A. Garcimartín, M. R. J. M. Pastor, C. Kähler, and I. Zuriguel, Don't get stuck!, https://doi.org/10.1103/APS.DFD.2016.GFM.V0053 (2016).

[18] A. Marin, H. Lhuissier, M. Rossi, and C. J. Kaehler, Clogging in constricted suspension flows, arXiv:1711.06119.

[19] J.-B. Salmon, L. Bécu, S. Manneville, and A. Colin, Towards local rheology of emulsions under couette flow using dynamic light scattering, Eur. Phys. J. E 10, 209 (2003). 
[20] S. Manneville, L. Bécu, and A. Colin, High-frequency ultrasonic speckle velocimetry in sheared complex fluids, Eur. Phys. J. Appl. Phys. 28, 361 (2004).

[21] J. Goyon, A. Colin, G. Ovarlez, A. Ajdari, and L. Bocquet, Spatial cooperativity in soft glassy flows, Nature (London) $\mathbf{4 5 4}$, 84 (2008).

[22] L. Isa, R. Besseling, A. N. Morozov, and W. C. K. Poon, Velocity Oscillations in Microfluidic Flows of Concentrated Colloidal Suspensions, Phys. Rev. Lett. 102, 058302 (2009).

[23] M. Sahimi and A. O. Imdakm, Hydrodynamics of Particulate Motion in Porous Media, Phys. Rev. Lett. 66, 1169 (1991).

[24] A. O. Imdakm and M. Sahimi, Computer simulation of particle transport processes in flow through porous media, Chem. Eng. Sci. 46, 1977 (1991).

[25] P. Bacchin, A. Marty, P. Duru, M. Meireles, and P. Aimar, Colloidal surface interactions and membrane fouling: Investigations at pore scale, Adv. Colloid Interface Sci. 164, 2 (2011).

[26] G. C. Agbangla, P. Bacchin, and E. Climent, Collective dynamics of flowing colloids during pore clogging, Soft Matter 10, 6303 (2014).

[27] P. Duru and Y. Hallez, A three-step scenario involved in particle capture on a pore edge, Langmuir 31, 8310 (2015).

[28] T. van de Laar, S. ten Klooster, K. Schroën, and J. Sprakel, Transition-state theory predicts clogging at the microscale, Sci. Rep. 6, 28450 (2016).

[29] U. Zimmermann, F. Smallenburg, and H. Löwen, Flow of colloidal solids and fluids through constrictions: Dynamical density functional theory versus simulation, J. Phys.: Condens. Matter 28, 244019 (2016).

[30] B. S. Zenamarkos and P. Bacchin, Colloidal jamming dynamics in microchannel bottlenecks, Langmuir 32, 1478 (2016).

[31] N. Y. C. Lin, C. Ness, M. E. Cates, J. Sun, and I. Cohen, Tunable shear thickening in suspensions, Proc. Natl. Acad. Sci. USA 113, 10774 (2016).

[32] M. E. Cates, K. Stratford, R. Adhikari, P. Stansell, J. C. Desplat, I. Pagonabarraga, and A. J. Wagner, Simulating colloid hydrodynamics with lattice boltzmann methods, J. Phys.: Condens. Matter 16, S3903 (2004).

[33] J.-C. Desplat, I. Pagonabarraga, and P. Bladon, Ludwig: A parallel lattice-boltzmann code for complex fluids, Comput. Phys. Commun. 134, 273 (2001).
[34] R. Adhikari, K. Stratford, M. E. Cates, and A. J. Wagner, Fluctuating lattice boltzmann, Europhys. Lett. 71, 473 (2005).

[35] S. P. Thampi, I. Pagonabarraga, and R. Adhikari, Latticeboltzmann-langevin simulations of binary mixtures, Phys. Rev. E 84, 046709 (2011).

[36] G. Bryant, S. R. Williams, L. Qian, I. K. Snook, E. Perez, and F. Pincet, How hard is a colloidal "hard-sphere" interaction?, Phys. Rev. E 66, 060501 (2002).

[37] N.-Q. Nguyen and A. J. C. Ladd, Lubrication corrections for lattice-boltzmann simulations of particle suspensions, Phys. Rev. E 66, 046708 (2002).

[38] K. Stratford, R. Adhikari, I. Pagonabarraga, and J. C. Desplat, Lattice boltzmann for binary fluids with suspended colloids, J. Stat. Phys. 121, 163 (2005).

[39] K. Stratford and I. Pagonabarraga, Parallel simulation of particle suspensions with the lattice boltzmann method, Comput. Math. Appl. 55, 1585 (2008).

[40] A. Garcimartin, D. R. Parisi, J. M. Pastor, C. Martin-Gomez, and I. Zuriguel, Flow of pedestrians through narrow doors with different competitiveness, J. Stat. Mech.: Theor. Exp. (2016) 043402.

[41] A. Garcimartin, J. M. Pastor, L. M. Ferrer, J. J. Ramos, C. Martin-Gomez, and I. Zuriguel, Flow and clogging of a sheep herd passing through a bottleneck, Phys. Rev. E 91, 022808 (2015).

[42] J. R. Valdes and J. C. Santamarina, Clogging: Bridge formation and vibration-based destabilization, Can. Geotech. J. 45, 177 (2008).

[43] K. To and H.-T. Tai, Flow and clog in a silo with oscillating exit, Phys. Rev. E 96, 032906 (2017).

[44] I. Zuriguel, D. R. Parisi, R. C. Hidalgo, C. Lozano, A. Janda, P. Alejandra Gago, J. P. Peralta, L. M. Ferrer, L. A. Pugnaloni, E. Clément, I. Pagonabarraga D. Maza, and A. Garcimartín, Clogging transition of many-particle systems flowing through bottlenecks, Sci. Rep. 4, 7324 (2014).

[45] J. M. Pastor, A. Garcimartín, P. A. Gago, J. P. Peralta, C. MartínGómez, L. M. Ferrer, D. R. Maza, D. Parisi, L. A. Pugnaloni, and I. Zuriguel, Experimental proof of faster-is-slower in systems of frictional particles flowing through constrictions, Phys. Rev. E 92, 062817 (2015). 